from gardens of species which are normally visited being entirely neglected for more attractive types. Upon the acuteness of this competition depends the importance to be attached to the sequence of flowering periods. Clements and I.ong, using the method of reciprocal bouquets, came to the conclusion that habit was the most important factor in determining preference, but that abundance of nectar or ease of access might overcome the effect of custom.

The marked capacity for memorisation by bees which training experiments reveal show that features of floral construction, of an apparently trivial character, may be of real importance in facilitating recognition. Moreover, the readiness with which bees discriminate the most profitable and easily worked flowers gives added significance to the minutiæ of floral architecture.

Further progress in the study of insect behaviour in relation to flowers would appear to demand the use of marked individuals of freshly hatched insects which have not acquired the habits that so largely determine their actions when adult.

\title{
X-ray Studies on the Crystal Structure of Iron and Steel.
}

$A T$ the recent meeting of the Iron and Steel InstiA tute Messrs. A. Westgren and G. Phragmen presented a continuation of work published by them two years ago at the same Institute. The present paper deals with the crystal structure of $\delta$ iron, the crystal shape of Cementite, and the structure of Austenite. In their previous paper a series of powder photograms of $\alpha, \beta, \gamma$ and $\delta$ forms of iron was reproduced. At that time all attempts to get a photogram of $\delta$ iron (stable only above $1400^{\circ} \mathrm{C}$.) in the pure state had failed. Its interference lines were mingled with those originating from $\gamma$ iron. By improved heating arrangements it has been possible to obtain a more uniform temperature, and the new photograms are quite free from $\gamma$ interferences and confirm the conclusion previously drawn, namely, that $\delta$ iron has the same lattice structure as $\alpha$ and $\beta$ iron.

The question as to how the atoms are grouped in Cementite $\left(\mathrm{Fe}_{3} \mathrm{C}\right)$ has even now not been solved. The powder photogram contains a very large number of lines, some of which flow into one another. As many of the interferences of this orthorhombic substance practically coincide it could not be settled definitely, without a more thorough experimental investigation, to which of the net planes the lines of the powder photogram correspond. To decide this question socalled "complete photograms" or " rotation photograms" have been taken of a Cementite crystal obtained from the blow-hole of a spiegel melt. The only developed faces (oor) were finely striated parallel to [oro]. The precision camera designed for this purpose was described by the authors in NATURE of January 26 , p. I22. The theory of the photograms obtained has been given by Schiebold and Polanyi. A special camera was constructed for establishing the lattice dimensions and the authors showed photograms thus obtained. The edges of the elementary parallelepiped are $4.5 \mathrm{I} 8 \AA, 5.069 \AA$, and $6 \cdot 736 \AA$. This corresponds with an axial ratio of $0.67 x: 0.753: x$. Laue photograms taken parallel with the three axes have given an axial ratio in perfect accordance with the above. If it is assumed that the elementary parallelepiped contains four molecules of $\mathrm{Fe}_{3} \mathrm{C}$ the density of Cementite must be $7 \cdot 68$. Values hitherto available range from 7.74 obtained by Benedicks to 7.59 by Levin and Dornhecker.

The discrepancy which has hitherto existed with regard to the crystal structure of Cohenite-the iron carbide present in meteorites-has been cleared up. Weinschenk, who first investigated this mineral, found that its composition corresponds to $\mathrm{Fe}_{3} \mathrm{C}$, but concluded that it probably belonged to the regular system. Goniometric measurements were afterwards carried out by Hussak on a Cohenite crystal obtained from a Brazilian meteorite. In spite of the small size of the crystal, the fact that its faces were frequently curved, and that most of them gave very poor reflections, Hussak concluded that Cohenite belonged to the regular holohedral system. Even so, some of his angles differed $2^{\circ}$ or more from the values characteristic of the cubic crystal. The authors show powder photograms of cementite and Cohenite which are practically identical. These two substances are therefore orthorhombic.

The authors have also used their precision camera to investigate the structure of Austenites obtained in various ways, both pure and intermixed with Martensite. They conclude that carbon dissolved in $\gamma$ iron has an enlarging influence on the lattice dimensions, and that the lattice parameter changes from $3.606 \AA$ in the case of Austenite containing 0.9 per cent. of carbon, to $3.629 \AA$ in the case of the saturated solution containing I. 7 per cent. The Martensite lines, however, are too cloudy and broad to enable conclusions to be drawn concerning the lattice dimensions of the $a$ iron in quenched steels. The authors reason that if carbon atoms replace iron atoms in the $\gamma$ iron lattice the dimensions should decrease with rising carbon content, since the carbon atoms are much smaller than those of iron. Since, however, the opposite is the case, it seems probable that Austenite is not formed by simple substitution, but represents an addition product with the carbon atoms in the interstices between the metal atoms.

Precision photograms of a manganese Austenitic steel, having a lattice parameter of $3.624 \AA$, show that in all probability this is the case. The density of the steel can be calculated either on the assump. tion that the iron and carbon atoms replace each other in one and the same lattice, or that the mass of the carbon atoms is uniformly distributed among the metal atoms which occupy the points of the facecentred cubic lattice. In the former case the density is $7 \cdot 36$, in the latter $7 \cdot 83$. The experimental value found was $7 \cdot 83$. The second assumption therefore is correct, and this Austenite is not formed by simple substitution but is an addition product. The real nature of the addition of carbon atoms cannot be decided on the basis of the results so far obtained. The authors suggest that it may be a substitution of the atoms of the $\gamma$ iron lattice by complexes consisting of an iron atom combined with one or more carbon atoms.
H. C. H. C.

NO. 2855 , vOL. I 147 